\title{
REVISTARACIÓN
}

Revista Educación

ISSN: 0379-7082

ISSN: 2215-2644

revedu@gmail.com

Universidad de Costa Rica

Costa Rica

\section{Factores que afectan la convivencia familiar: análisis micro etnográfico a través de juegos tradicionales en un grupo social en México}

Coyotecatl Xochimitl, Luz María; Murrieta Ortega, Raymundo

Factores que afectan la convivencia familiar: análisis micro etnográfico a través de juegos tradicionales en un grupo social en México

Revista Educación, vol. 46, núm. 1, 2022

Universidad de Costa Rica, Costa Rica

Disponible en: https://www.redalyc.org/articulo.oa?id=44068165018

DOI: https://doi.org/10.15517/revedu.v46i1.43829

\section{(c) (1) $\Theta \Theta$}

Esta obra está bajo una Licencia Creative Commons Atribución-NoComercial-SinDerivar 3.0 Internacional. 


\title{
Factores que afectan la convivencia familiar: análisis micro etnográfico a través de juegos tradicionales en un grupo social en México
}

\author{
Factors Affecting Family Coexistence: A Microethnographic Analysis Using Traditional Games in a Mexican Social \\ Group
}

Luz Maria Coyotecatl Xochimitl

Telesecundaria Simbolos Patrios, México

luzmariacx89@gmail.com

https://orcid.org/0000-0001-6864-5188

Raymundo Murrieta Ortega
Benemérito Instituto Normal del Estado "Gral.Juan
Crisóstomo Bonilla", México
murrieta.ortega.r@bine.mx

https://orcid.org/0000-0003-4856-9573
DOI: https://doi.org/10.15517/revedu.v46i1.43829

Redalyc: https://www.redalyc.org/articulo.oa? $\mathrm{id}=44068165018$

Recepción: 19 Octubre 2020

Aprobación: 22 Febrero 2021

\section{Resumen:}

¿Qué factores afectan la convivencia familiar? A partir de esta interrogante se realiza un estudio cualitativo con diseño micro etnográfico (Creswell, 2005), con el objetivo de analizar los aspectos no favorecedores de la convivencia entre un grupo de estudiantes que cursa el quinto grado en una escuela primaria y sus respectivas madres y padres de una comunidad de la región centro de México. Para la obtención de los datos se emplearon dos tipos de observación: sistemática y participante; además de la entrevista, la encuesta (cuestionarios con preguntas abiertas para padres, madres y alumnado), fichas de juegos y el diario de campo. La información obtenida fue analizada a través de la codificación directa y axial, que se integró como unidad de análisis la convivencia entre padres y madres con hijos e hijas, y cinco categorías surgidas desde el abordaje inductivo. El estudio se sustenta en la teoría sociocultural de Vygotsky (1933, citado en Meece, 2001), es decir, aprender a través de la interacción, en la teoría de la dinámica infantil de Buytendijik (1935) y en la teoría del excedente energético de Herbert Spencer (1855, estás últimas teorías citadas en Gallardo-López y Gallardo-Vázquez, 2018). El uso de juegos tradicionales como recurso pedagógico en la clase de educación física contribuyó a establecer un vínculo de trabajo en la escuela y en casa entre madres, padres, hijas e hijos, además de favorecer la interacción familiar y el gasto energético preferentemente en la población preadolescente. En el estudio se utilizaron diferentes juegos tradicionales de México; se finalizó con una feria denominada Los juegos de mi abuelo. Los resultados dan a conocer que los factores que afectan la convivencia entre quienes integran la familia del grupo en estudio son la escasa comunicación, limitado uso del tiempo libre, indiferencia por el aprendizaje, responsabilidad laboral por parte de las madres y los padres, y uso excesivo de aparatos electrónicos de las personas preadolescentes.

Palabras ClaVe: Juegos tradicionales, Convivencia, Micro etnografía, Educación física, Familia.

\section{Abstract:}

A qualitative study was conducted to determine factors that impacted family coexistence. The study used a microethnographic design (Creswell, 2005) to examined aspects that did not benefit coexistence among a group of fifth grade students in Central Mexico and their parents. Data was collected through interviews, systematic and participant observation, a survey, which included open questions both for parents and students, a game card and a field diary. The data obtained was analyzed through direct and axial coding which included parent-child coexistence as a unit of analysis according to five categories arising from an inductive approach. The study is based on the sociocultural theory of Vygotsky (1933, cited in Meece, 2001) on learning through interaction, Byutendijik's (1935) theory of child dynamics and the energy surplus theory by Herbert Spencer (1855, the latter cited in Gallardo-López and Gallardo-Vázquez, 2018). Use of traditional games as a pedagogical resource during P.E. class, served to strenghten the bond between parents and their children. It also promoted greater family interaction and energy expenditure, particularly among preteenagers. Various traditional Mexican games were used in the study, concluding with a fair called Games Played by My Grandfather. Some of the factors that were found to negatively impact the coexistence of the family group studied 
include poor communication, limited use of free time, indifference to learning, Parent responsiblities at work and excessive use of electronic devices.

KeYworDs: Traditional games, Coexistence, Microethnography, Physical Education, Family.

\section{INTRODUCCIÓN}

En la actualidad existe reducido tiempo para que las personas integrantes de la familia convivan de forma lúdica, esto se corrobora a partir de la experiencia personal de radicar en una comunidad rural e interactuar día con día con las personas que la habitan, en donde se pudo observar que los niños y las niñas de ahora dedican tiempo excesivo al uso de los medios tecnológicos desde edades tempranas. Por su parte, los padres y madres de familia se enfocan al trabajo y esto, generalmente, evita que puedan relacionarse en ambientes lúdicos, pues se limita, en cuestiones de tiempo, la interacción con sus hijos e hijas.

Es por ello que la intención del estudio se enfoca en analizar los aspectos que dificultan la convivencia entre el estudiantado de quinto grado de una primaria ubicada en una comunidad del estado de Puebla en México, y sus respectivos padres y madres. Lo anterior porque se percibe que los niños y las niñas en la actualidad han incorporado nuevas formas de relacionarse y de ocupar su tiempo libre. Es muy común observar que se divierten con un aparato tecnológico: el smartphone ha pasado a ser un juguete de moda; juegan con él en el salón, en las escaleras o el patio de la escuela.

En consecuencia, surge la interrogante: ¿qué pasa con los juegos de antes, donde los niños y niñas jugaban con sus compañeros del colegio, amigos, vecinos, familia y se divertían con infinidad de actividades lúdicas y no sólo con un objeto? Actualmente los niños y las niñas tienen pocas oportunidades para jugar con sus padres y madres, y la escuela no establece proyectos educativos que fomenten las actividades lúdicas en familia, o bien, al implementarlas, los padres y madres de familia no disponen de tiempo para asistir al centro educativo a interactuar con sus hijos e hijas.

Por lo anterior, es necesario que los niños y las niñas desarrollen nuevas formas para interactuar con las demás personas; precisamente, la familia y la escuela son los escenarios ideales para promover ese desarrollo. La familia es la parte esencial en el desarrollo físico-motor-cognitivo-social del niño y de la niña; sin embargo, generalmente los padres o madres de familia se preocupan más por las cosas materiales y no por las emocionales; esto provoca el descuido en la educación de sus hijos o hijas, pues en muchas ocasiones prefieren regalarle un smartphone, que convivir con la familia. Este desapego provoca que los hijos o hijas prefieren estar con el teléfono que con sus familiares y todo esto repercute en la conducta aislada o agresiva de la población infantil en casa y en la escuela.

Al mismo tiempo, se ha observado que el alumnado que cursa quinto grado de primaria, cuya edad oscila entre 11 y 12 años, llega solo a la escuela y se va solo o con sus amistades. Se identifica que poco a poco se va creando un distanciamiento entre madres y padres de familia e hijos o hijas, debido a, que, por la edad de las personas estudiantes, existe la idea de autosuficiencia y autocuidado, incluso esa misma idea la perciben los padres o madres de familia, quienes tienen la noción de que a esa edad se cuidan solos y solas.

Las familias, en el contexto de la comunidad en estudio, son de un nivel socioeconómico medio bajo, de acuerdo a las estadísticas que se obtuvieron en la dirección escolar; la ocupación de los padres y madres de familia es variada, un porcentaje amplio de madres de familia desempeñan labores de amas de casa y la manutención familiar preferentemente está a cargo de los padres. La ocupación predominante es la albañilería, el comercio y la agricultura, pues una mayoría se dedica a trabajos en el campo, puesto que en la comunidad siembran maíz y otros vegetales; en cuanto al nivel de escolaridad de los padres y madres de familia, se identifica que pocos concluyeron la primaria y la mayoría no sabe leer ni escribir.

De acuerdo a la experiencia, se observa cómo los padres y, especialmente, las madres de familia experimentan situaciones adversas en la educación de sus hijas e hijos, hasta solicitar apoyo con las instancias correspondientes ante la rebeldía y comportamiento inapropiado de sus hijos o hijas preadolescentes, tanto 
en la escuela como dentro del seno de la familia. En ocasiones, se realizaban afirmaciones acerca de que los hijos o hijas manifestaban rebeldía. Lo anterior, afectaba el desarrollo social de los niños y niñas, es decir, su capacidad para interactuar y relacionarse con las demás personas.

Por lo anterior, se establecen los siguientes cuestionamientos:

- ¿Cuáles son los factores que afectan la convivencia entre el alumnado de quinto grado de primaria de una escuela perteneciente a una comunidad de la región centro de México y sus padres y madres?

- ¿Cómo es la convivencia entre alumnos y madres padres de familia del grupo de quinto grado de una escuela primaria de la región centro de México, a partir de lo observado al inicio y cierre de la jornada escolar?

- ¿Qué efecto tuvo la aplicación de juegos tradicionales en el fortalecimiento de la convivencia en un grupo de una escuela primaria de la región centro de México y sus padres y madres?

\section{ANTECEDENTES}

Sobre el tema de la convivencia familiar, en la actualidad han surgido cambios drásticos en ella; la influencia de la sociedad, la tecnología y otros factores la han transformado. Murillo (2009) describe que los cambios de la sociedad influyen en la función de la familia, donde existe un menor compromiso con la formación de sus hijos e hijas debido a las diferentes tareas de las madres y los padres, las cuales ocasionan que sean personas más permisivas.

La influencia de la tecnología en la vida diaria de las familias, tanto en el contexto urbano como en el rural, ha provocado que las personas estudiantes tengan más cosas materiales y le dediquen más tiempo a su uso, pero esto trae como consecuencia que las niñas y los niños pasen menos tiempo con sus padres o madres.

Murillo (2009) describe que el principal grupo social es la familia, debido a que es con quien conlleva las actividades de la vida cotidiana, transmite costumbres y moldea comportamientos. En el contexto de la investigación, la familia representa el ejemplo de comportamiento y de actividades que las personas estudiantes realizan en la vida diaria.

Por otra parte, en su trabajo de investigación, De León (2011) afirma que existen dos grandes instituciones que contribuyen a una construcción ciudadana de la población infantil: la escuela y la familia. Pero argumenta que ambas instituciones no pueden trabajar por separado; al contrario, deben desempeñarse de forma unida para lograr la edificación de una mejor ciudadanía. Desde esta perspectiva, el presente estudio analiza este distanciamiento entre escuela y familia, al proponer formas de actuación lúdicas en beneficio del uso del tiempo libre del grupo de preadolescentes.

En este orden de ideas, se constata que la escuela no debe trabajar sola, al no considerarse el único escenario educativo, pues se afirma que la familia y los medios de comunicación representan una relevante función educativa. Al mismo tiempo, se destaca que la escuela por sí sola no es suficiente para aportar los elementos necesarios para la formación ciudadana de infantes, sino que se sugiere la participación de las madres y los padres como agentes colaboradores de la escuela para la formación integral de sus hijas e hijos (Ortiz, 2011, citado por De León, 2011). Por lo tanto, se considera que ambas instancias tienen la función común y el mismo propósito de educar y formar a la ciudadanía.

Hernández y Carrión (2019) refieren que las interacciones en la familia y en la escuela han sufrido modificaciones con la aparición de la tecnología, de manera que ambas instituciones se convierten en espacios de encuentro para las nuevas representaciones de la integración social. El uso de la tecnología en el contexto rural tiene un papel de entretenimiento, lo que provoca en el alumnado un uso excesivo y poco provechoso.

Algo semejante establecen Lillo y Leiva (2012), quienes argumentan que se deben promover interacciones entre la escuela y la familia, a fin de prevenir propuestas encontradas y propiciar un trabajo vinculado que disminuya los postulados conductuales que se sugieren en la escuela con los que se proponen desde la familia. 
Por ese motivo, se afirma que la tríada familia, escuela y docente representa un papel clave para promover valores que pueden influir en el factor convivencia.

Un grupo de estudiosos (Ramírez-García et al., 2018), en su investigación, describen que "la introducción de Internet y los dispositivos móviles, como el Smartphone en los hogares, ha tenido una gran repercusión en el seno familiar, modificando muchos de los hábitos comunicativos y relacionales establecidos hasta el momento" (p. 137). Por lo tanto, sugieren que las madres y padres deberán poseer una serie de "competencias parentales positivas en torno al cuidado y protección del menor, la orientación y organización doméstica, el reconocimiento del menor, la educación, la agencia personal y la competencia para toda la vida". (RamírezGarcía et al., 2018, p.137).

Es por todo lo anterior que, desde el presente trabajo, se propone que las familias estimulen la parentalidad positiva. Loizaga (2011) describe las bondades de la parentalidad positiva en tres niveles: 1) las bases de la parentalidad que deben aportar las familias, 2) el comportamiento parental positivo, y 3) el metalenguaje o función reflexiva de la parentalidad. La base de la parentalidad positiva es la psicología positiva; esta última establecida por Seligman (2017), que argumenta la necesidad de experimentar emociones positivas en las diferentes etapas para el desarrollo de una vida plena. Por lo anterior, es importante el uso del juego para crear un ambiente propicio para las relaciones sociales positivas entre padres, madres, niños y niñas.

Loizaga (2011), en su propuesta, destaca que en el primer nivel las bases de la parentalidad consisten en los procesos, orientaciones y ejemplos que el grupo de infantes necesita de las figuras parentales para desarrollarse como personas; el segundo nivel, comportamiento parental positivo, hace referencia a las acciones y procesos para crear vínculos positivos entre padres e hijos, una de las acciones que propone es "jugar y disfrutar con los hijos" (Loizaga, 2011, p.49); el tercer nivel, metalenguaje de la parentalidad, es la función reflexiva parental que realizan los integrantes de la familia para dar sentido a la vida desde una visión ética y espiritual.

Con base en los argumentos de Megías y Elzo (2006), se valora un mayor nivel de responsabilidad de la figura docente en relación a la educación del estudiantado. Lo anterior porque el escenario social actual, en el que se desenvuelven las familias, influye evidentemente en el tiempo que el alumnado está en la escuela con el profesorado, debido a las jornadas de trabajo más amplias de las madres y padres para cubrir las necesidades de quienes integran la familia.

Por tanto, la educación de las niñas y los niños queda en manos de dos importantes instituciones: la familia y la escuela; esto compromete a ambas instancias a vincularse colaborativamente para transmisión de normas y valores que incidan en la formación de una ciudadanía con valores.

\section{Marco teórico}

El soporte teórico de la investigación se sustenta en los postulados de Vygotsky (citado en Meece, 2001) desde la perspectiva del desarrollo cognoscitivo y sociocultural; en la propuesta de Spencer (como se citó en Gallardo-López y Gallardo-Vázquez, 2018) con la temática del juego que hace referencia al excedente de energía; y la dinámica infantil de Buytendijk (como se citó en Gallardo-López y Gallardo-Vázquez, 2018), que establece que la niña y el niño juegan por naturaleza. También se aborda el estudio con la perspectiva del apego de Bowlby (1986) y en la de los estilos parentales (parentalidad positiva) conceptualizados por Loizaga (2011); ambas se fundamentan en la psicología positiva, propuesta por Seligman (2017), para fomentar el vínculo afectivo entre madres, padres, hijas e hijos.

La teoría del desarrollo cognoscitivo de Vygotsky (citado por Meece, 2001) destaca las interacciones de las personas con su entorno social, al afirmar "que no es posible entender el desarrollo del niño si no se conoce la cultura donde se cría" (p. 217). Ahora bien, las personas adultas tienen la encomienda de transmitir sus conocimientos a las más jóvenes para estimular el desarrollo intelectual; de acuerdo con esta teoría, la experiencia vivida por cada ser humano, así como su historial cultural en la etapa infantil, son elementos 
indispensables para entender el desarrollo cognoscitivo y de ahí partir para obtener resultados favorables en su proceso de enseñanza aprendizaje.

Desde la teoría sociocultural de Vygotsky (citado en Meece, 2001), se retoma el planteamiento acerca de cómo el niño y la niña aprenden de forma social, en una participación activa en el ambiente que les rodea. Específicamente, el niño o la niña desarrolla su aprendizaje mediante la interacción social, en la que va adquiriendo nuevas habilidades. Por ello, Vygotsky (como se citó en Meece 2001) afirma que:

Los adultos guían y apoyan el desarrollo intelectual del niño. A través de la supervisión social que le ofrecen, puede funcionar en un nivel superior del desarrollo, es decir, en la zona del desarrollo proximal. Algunos investigadores han estudiado los procesos con que el adulto guía la intervención en la zona. Analizaremos dos conceptos distintos, pero estrechamente conexos de este proceso social: la participación guiada y el andamiaje (asistencia). (p. 134)

Por otro lado, el andamiaje se considera aquel apoyo temporal de la persona adulta durante su desarrollo; este consiste en guiarlo y posteriormente le permite "al niño asumir gradualmente mayor responsabilidad en la administración de la actividad” (Meece, 2001, p. 134).

El estudio también se fundamenta en la teoría del excedente de energía, propuesta desde 1855 por Spencer (como se citó en Gallardo-López y Gallardo-Vázquez, 2018). Se destaca que el individuo acumula grandes cantidades de energía, las cuales estaban anteriormente dedicadas a la supervivencia. Desde la perspectiva de Spencer, estas grandes cantidades de energía sobrante deben ser liberadas en diversas actividades sin una finalidad inmediata, para así evitar tensiones al organismo; de manera que el juego, junto con las actividades artísticas y estéticas, se convierten en una de las principales herramientas que tiene el cuerpo humano para un desarrollo integral.

Por tanto, la teoría de Spencer (como se citó en Gallardo-López y Gallardo-Vázquez, 2018) se apoya en la idea de que la infancia es una etapa del desarrollo en la que el niño y la niña no tiene que realizar ningún trabajo para poder sobrevivir, dado que sus necesidades se encuentran cubiertas por la intervención de sus padres o madres, y elimina el excedente de energía a través del juego, actividad que ocupa los grandes espacios de tiempo que le quedan libres.

El estudio también se basa en la teoría de la dinámica infantil establecida en 1935 por Buytendijk (como se citó en Gallardo-López y Gallardo-Vázquez, 2018), que interpreta el juego como una actividad derivada de una actitud o dinámica infantil. Dicha teoría se fundamenta en la idea de que el juego depende de la dinámica infantil, y que un niño y la niña juegan porque son infantes; es decir, que los caracteres propios de su 'dinámica' le impulsan a no hacer otra cosa que jugar. Por ello, se valora importante aprovechar esta etapa de la vida del niño y la niña para que los padres o madres de familia asuman una actitud o dinámica infantil que contribuya con su participación al desarrollo integral de sus hijos e hijas.

La investigación considera la perspectiva de Bowlby (1986), quien argumenta desde la teoría del apego que "los seres humanos de todas las edades son más felices y pueden desarrollar mejor sus capacidades cuando piensan que, tras ellos, hay una o más personas dignas de confianza que acudirán en su ayuda si surgen dificultades" (p. 128). Para ello, el vínculo afectivo son aquellas acciones que promueven tener un acercamiento y establecer confianza con una persona o varias, siempre y cuando sean cercanas. Se ajustan estos planteamientos al estudio con padres o madres e hijos o hijas.

Aunado a lo anterior, desde la teoría de los estilos parentales se analiza la propuesta de parentalidad positiva, la cual surge de la psicología positiva (Seligman, 2017) y que conceptualiza Loizaga (2011) en tres niveles: factor primordial para el adecuado desarrollo cerebral, emocional y social de infantes. Destaca que "el desarrollo de los tres niveles hace que los menores y las personas estructuren su mente, su lenguaje, sus relaciones interpersonales y su yo de manera armoniosa y madura, lo cual les ayuda a convertirse en adultos sanos" (Loizaga, 2011, p.70).

Sobre la temática de estilos parentales, Rodrigo (2015) argumenta que: 
Un padre positivo es el que atiende, potencia, guía y reconoce a sus hijos como personas con pleno derecho. La parentalidad positiva no es permisiva y requiere de la implementación de los límites necesarios para que los niños puedan desarrollarse plenamente (p. 21).

\section{Metodología}

\section{Investigación cualitativa con diseño micro etnografía}

El abordaje de la investigación se realizó desde una perspectiva cualitativa, dado que el estudio estuvo orientado a comprender la realidad social en su contexto natural para interpretar fenómenos como la interacción entre madres, padres, hijas e hijos. Al mismo tiempo, tuvo un diseño micro etnográfico (Creswell, 2005) porque se centró en una situación social concreta.

Sobre este tema, se afirma (Hernández et al., 2014) que este tipo de diseños "pretenden explorar, examinar y entender sistemas sociales (grupos, comunidades, culturas, sociedades)” (p. 482). Por otra parte, especialistas (Rodríguez et al., 1999) describen el método etnográfico como el método de investigación por el que "se aprende el modo de vida de una unidad social concreta" (p. 44), donde ésta puede ser "una familia, una clase o un claustro de profesores" (Rodríguez et al., 1999, p. 45) de una escuela.

Por tanto, la población objeto de estudio fue un grupo de quinto grado de primaria conformado por 29 estudiantes (10 niñas y 19 niños) de entre 10 y 11 años de edad y sus respectivos padres y madres (38). La muestra fue perteneciente a una comunidad del estado de Puebla, México. McMillan y Schumacher (2011) afirman que en este momento la persona investigadora "toma sujetos que resultan accesibles o que pueden representar ciertos tipos de características. Por ejemplo: una clase de estudiantes” (p. 140). Por lo tanto, la investigación se realizó en una escuela primaria ubicada en uno de los 217 municipios que conforman el estado de Puebla, México.

\section{Instrumentos para la recolección de datos}

Para recolectar los datos se utilizaron los siguientes medios: la observación sistemática y participante, la encuesta, las fichas de juego, la entrevista y el diario de campo.

Cabe destacar que una acción primordial fueron las tareas asignadas para realizar en casa. Después de aplicado el juego tradicional en la sesión de educación física, se le solicitaba al alumnado compartirlo con sus familias, para ello se les pedía el llenado de una ficha para valorar los resultados. La ficha contenía las siguientes partes: a) Nombre del juego, b) Edad, c) Material, d) Objetivo, e) Esencia del juego, f) Desarrollo, g) Variantes, h) Nombre del alumno, i) ¿Con quién lo jugarías? Ver Figura 1. 




FIGURA 1.

Ficha de juegos tradicionales

Fuente: elaboración propia.

La observación participante fue de gran utilidad durante la feria de juegos tradicionales, evento en donde participaron los padres y madres de familia. Rodríguez et al. (1999) definen a la observación participante como el "método interactivo de recogida de información que requiere una implicación del observador en los acontecimientos o fenómenos que está observando” (p. 165). Es decir, que interviene y observa a la vez, tal y como afirman: "permite al investigador desempeñar el doble rol de investigador y participante" (Rodríguez et al.,1999, p.165).

También se utilizó la encuesta mediante el diseño dos cuestionarios, uno aplicado a los padres y madres de familia y otro al alumnado. De las madres y padres se obtuvo información como el tipo de familia, nivel académico, tipo de vivienda, entre otros. Los resultados de la encuesta fueron de utilidad para identificar en qué familias existe distanciamiento, conflictos o falta comunicación. Además, se pudo ratificar que las madres de familia están en claro acuerdo con la falta de comunicación que hay en sus familias; según las preguntas realizadas, es muy importante rescatar que, después de aplicado el instrumento y mediante la sensibilización de la importancia de la interacción entre madres, padres, hijas e hijos, comenzaron a apoyar y jugar con sus hijos e hijas.

También se aplicó un cuestionario al alumnado para determinar si conocían todo lo referente a los juegos tradicionales. Se realizó primero un juego dentro de la sesión de Educación Física con el fin de observar si sabían jugarlo, puesto que en el quinto bloque de cuarto grado del Plan de Estudios 2011 de la Secretaría de Educación Pública [SEP] se trabaja con juegos tradicionales y autóctonos. De manera interpretativa se dio el conocimiento sobre el juego del avión, una parte del estudiantado no sabía cómo se jugaba, otra sí, pero lo más sorprendente es que ya tenía mucho tiempo de que no lo practicaban (Secretaría de Educación Pública, 2011).

Además, se aplicaron entrevistas por grupos focales a los padres de familia durante la reunión de información de la propuesta de investigación y después de la feria de juegos tradicionales.

\section{Análisis de los datos}

El abordaje de la investigación se realizó desde una perspectiva inductiva, es decir "sumergiéndose literalmente en un documento o situación para identificar los temas o las dimensiones más relevantes" (Massoi et al., 2009, p. 359). Posterior a lo mencionado, surgieron cinco categorías.

Para realizar el análisis de la información se retomaron las propuestas metodológicas de Massoi et al. (2009) y de Álvarez-Gayou (2003), debido a que sus propuestas se refieren a un análisis manual y estructurado 
de la información recopilada. Massoi et al. (2009) establecen dos pasos para el análisis de la información, el primero implica "seleccionar, focalizar y abstraer los datos brutos en unidades de significado que se denominan categorías de contenido, de acuerdo con unos criterios temáticos determinados” (p. 358). Las autoras argumentan que "esta idea resume el procedimiento fundamental del análisis de datos cualitativos que consiste en buscar temas comunes o agrupaciones conceptuales en un conjunto de narraciones recogidas como datos de base o de campo" (Massoi et al., 2009, p. 358).

Asimismo, el segundo se enfoca en la codificación y categorización. Con respecto a la categorización "entendida como la división y simplificación del contenido a unidades o categorías de acuerdo con criterios temáticos", por otra parte, la codificación es la "operación concreta por la cual se asigna a cada unidad de contenido él código propio de la categoría donde se incluye” (Massoi et al., 2009, p. 358).

Strauss y Corbin (1988 citados en Álvarez-Gayou, 2003) afirman que existen dos formas de codificar: abierto y axial. Los códigos abiertos consisten en identificar y señalar palabras o porciones del texto importantes; mientras que en la codificación axial se retoman los códigos abiertos y se procede a relacionarlos entre sí con el objetivo de establecer una explicación entre ellos. Para el análisis de la información, ÁlvarezGayou (2003) propone los siguientes pasos:

1. Digitalizar la información de los diarios, cuestionarios y entrevistas.

2. Imprimir la información (tenerla en papel) utilizando diferentes colores en la impresión. Dejar un amplio margen del lado derecho para hacer anotaciones. Tener marcadores de color, hojas grandes de cartulina, tijeras, lápiz adhesivo, borrador, plumón negro y bolígrafo o lápices.

3. Lecturas repetidas del material, para identificar palabras o ideas que se repiten; con el marcador de color señalar porciones de texto que llamen la atención (codificación abierta). Con marcador de otro color se subrayan partes del texto que podrían servir de citas textuales (citas de diario o de entrevista).

4. Codificar los datos con etiquetas (palabras que engloben la idea del mensaje). Una vez colocados los códigos o categorías que se pusieron en el margen derecho y se utilizará una cartulina para cada una de ellas, donde se colocará el nombre del código en la parte superior. Pegar la cartulina en la pared.

5. Recortar cada porción del texto y pegarlo en la cartulina correspondiente al código.

6. Surgimiento de nuevas categorías o subcategorías: en las cartulinas ya se observaron las diferentes categorías y lo que incluyen. Es factible que, al ver todo el material desplegado, la persona que analiza se dé cuenta de que puede abrir nuevas categorías y así pondrá una nueva cartulina.

7. A partir de esto se inicia el proceso de codificación axial y el desarrollo de una primera versión del reporte final de la investigación.

\section{Resultados}

La lógica inductiva utilizada en la investigación permitió el surgimiento de la dimensión o unidad de análisis principal que es la convivencia entre padres, madres, hijos e hijas, a partir de cinco categorías o factores que afectan su convivencia, las cuales son descritas a continuación: 1) insuficiente comunicación entre padres, madres, hijos e hijas; 2) limitado uso del tiempo libre juntos; 3) escaso interés de los padres por el rendimiento escolar de sus hijos o hijas; 4) responsabilidad laboral y 5) uso excesivo de aparatos electrónicos.

\section{1) Insuficiente comunicación entre padres, madres, hijos e hijas}

Los datos obtenidos reflejan la escasa comunicación que se manifiesta entre padres o madres e hijos o hijas del grupo de estudio. En la actualidad, las familias muestran desapego y escasa comunicación, esto se pudo 
percibir durante la entrevista en grupos focales con padres y madres de familia al finalizar la feria de juegos tradicionales, en donde se observó e interpretó que existe comunicación reactiva (gritos) y no asertiva, como es recomendable; además de machismo, reflejado generalmente en las expresiones de los padres cuando afirman que las niñas no deben estudiar por que las van a mantener; que son mujeres y sus esposos no las van a dejar trabajar; solo con que aprendan a escribir es más que suficiente; ellas solo sirven para que realicen las labores del hogar y sirvan de comer. Tales afirmaciones provocan focos de alarma en torno a las creencias de las personas participantes.

Dentro del aspecto de la comunicación en el cuestionario realizado al alumnado, se destaca que los hijos o hijas no tienen confianza en los padres o madres de familia (y si la tienen es con aquella persona con la que convive más). En las palabras del alumnado se refleja que a la mamá no le tienen confianza porque siempre los regaña por lo que hacen, además no les limita en sus amistades. Por otro lado, se identifica que al papá sí le tienen confianza, porque los escucha, les da lo que quieren, consejos y les ayuda en la tarea. Pero al mismo tiempo, existe la contraparte acerca del reconocimiento a la labor de la madre cuando comentan que la mamá es a la que le tienen confianza, porque además de ayudarles en su aprendizaje, les cuida y protege, además de que siempre les pregunta cómo les fue en la escuela o si tienen amigos; por otro lado, afirman que al padre casi no lo ven y cuando quieren contarle algo, muchas veces llega cansado o simplemente se les olvida.

Por tanto, en la búsqueda de información, el Sistema Estatal de Desarrollo Integral de las Familias (Desarrollo Integral de la Familia, 2020) propone temáticas para promover la comunicación en la familia, al recomendar a los padres acerca de buenos hábitos para las acciones correctivas, sin transgredir su integridad personal, es decir, por medio de acciones que no ofendan o agredan; además, recomienda el uso de vocabulario apropiado. Por tanto, se puede ser un padre o madre de familia que utiliza acciones correctivas de manera firme, pero sin lastimar a los niños o a las niñas. Si las personas adultas son conscientes de reprender a sus hijos o hijas de manera pacífica, su influencia formativa sobre será mayor .

\section{2) Limitado uso del tiempo libre compartido}

Los padres y madres de familia no reconocen la importancia de aprovechar el tiempo libre con sus hijos o hijas, y desafortunadamente, carecen de oportunidades de utilizarlo de forma provechosa.

$\mathrm{Al}$ mismo tiempo, a la mayoría de las madres y padres de familia les preocupa lo que hacen sus hijos o hijas en el tiempo libre. La población infantil debe aprender a aprovecharlo de manera constructiva, para evitar en todo lo posible los riesgos que pueden presentarse, como son el aburrimiento, los accidentes, la vagancia, la delincuencia y la drogadicción. No obstante, el tiempo libre que comparten los padres, madres con sus hijos o hijas se ve limitado por diferentes factores como la responsabilidad laboral, el interés de ambas partes por la tecnología (TV y smartphone) y otras aficiones.

Hay muchos y muy diversos caminos para enseñarles a disfrutar de su tiempo libre de forma constructiva para una vida productiva y saludable, por ejemplo, participar en infinidad de actividades lúdicas, interactuar con la familia en paseos, juegos de mesas, juegos tradicionales o tocar instrumentos musicales.

\section{3) Escaso interés de los padres o madres por el rendimiento escolar de sus hijos o hijas}

Dentro de la familia, según las respuestas de las madres y padres que contestaron el cuestionario, hay muchos factores que inciden de forma negativa en la formación de sus hijos e hijas. Un factor clave es el poco tiempo que las madres y padres están en casa al pendiente de las tareas de su hijo o hija; otro factor influyente es el cansancio que manifiestan al llegar a casa y poco interés por supervisar el desempeño en la escuela de sus hijos e hijas 
Una de las características que más se destaca en el grupo infantil es que no obtienen apoyo de sus padres o madres en las tareas escolares y la desmotivación. A los niños y niñas les afecta el poco interés de sus padres o madres por rendimiento académico; manifiestan que su situación personal no es la ideal, debido a los problemas que viven en casa, lo que provoca que no presten atención a las clases, esto gradualmente contribuye a que disminuya su rendimiento escolar.

La encuesta aplicada a los padres y madres de familia mediante un cuestionario, confirmó que en algunas ocasiones este grupo no asiste a los llamados de la escuela debido a que el personal docente no da información valiosa o se les hace tarde para llegar a las reuniones convocadas.

Cabe destacar que la mayoría de las madres generalmente asisten a las actividades de la escuela, los padres se dedican a trabajar; en ese mismo sentido, son las madres las que casi siempre preparan el desayuno a sus hijos e hijas para ir a la escuela, aunque algunas mamás mencionaron que sus hijos e hijas ya están grandes y que pueden prepararse su propio desayuno, o en su defecto, les proporcionan dinero para que se compren alimentos en la escuela.

Con respecto al afecto y cariño, algunas madres de familia sí dan abrazos y besos, la mayoría los regañan mucho porque no atienden sus tareas con prontitud, y pocas comentan que sus hijos o hijas ya les da pena que hagan eso enfrente de sus compañeritos y compañeritas. No obstante, solo las mamás saben cómo se comporta su hijo o hija, puesto que los padres casi no los ven debido a la responsabilidad laboral.

También se observó que algunos alumnos o alumnas se les dificultaba expresarse (los que eran tímidos o tímidas), también había alumnado que realizaba alguna maldad (una broma o simplemente realizar un golpe con el material en práctica). Debido a lo anterior, en la feria de juegos tradicionales, en donde hubo interacción familiar, se puso énfasis en observar la conducta de las personas que integran la familia, para verificar si la manera de comportarse del estudiantado es la misma o cambia con la presencia, ya sea de la madre o del padre de familia; se corroboró que varía solo un poco.

Otro aspecto importante es la comparación que los padres y madres establecen entre sus hijos o hijas, muchas veces sin darse cuenta lo hacen e inconscientemente emiten mensajes sobre que uno de sus hijos o hijas estudia o trabaja y la otra persona no.

\section{4) Responsabilidad laboral de los padres o madres de familia}

Como puede observarse en la investigación, existen dos instancias importantes que determinan el actuar de cada una de las personas: la familia y su trabajo. Dentro de la observación, al preguntarle al alumnado sobre aspectos de su familia, se consideró que el padre, generalmente, se dedica al trabajo en el campo durante jornadas laborales que inician desde muy temprano, por lo que no conviven mucho tiempo con sus hijas e hijos (pues llega cansado y lo único que quiere es comer y descansar). Por otro lado, la mamá se dedica a la educación y a atender a su esposo, hijos e hijas (darle de comer, preparar el itacate para ir al campo, lavarle y hacer labores del hogar). Ahora tienen que trabajar tanto los padres como las madres en turnos laborales a veces alargados excesivamente (otro factor más para no convivir con sus hijas e hijos).

\section{5) Uso excesivo de aparatos electrónicos}

A la pregunta hacia los niños y niñas: “¿Qué hacen en su tiempo libre?”, la respuesta de una gran parte fue que ven televisión o utilizan su smartphone. Lo anterior es un reflejo de la realidad en el entorno familiar, escolar y social, esto provoca que se cuestione si el uso de la tecnología es factor influyente en la falta de comunicación y convivencia en la familia. Por un lado, se debe valorar los aparatos electrónicos como importantes medios de comunicación, por ejemplo, cuando diferentes integrantes de la familia se conectan a través del celular para 
saber dónde están y cómo se encuentran mientras (los padres o madres) trabajan, o para pedir un permiso y estar al pendiente.

Con referencia a lo anterior, se describe lo que pasó en la entrevista que se realizó a los padres y madres de familia. Se concluyó que los niños y niñas que más ven televisión, están con el smartphone, juegan en el $X b o x$, entre otros aparatos tecnológicos, tienen repercusiones como realizar menos actividad física, poca convivencia con sus amistades, desinterés por interactuar con su mamá o papá y hacer la tarea.

El uso excesivo de la tecnología es una situación difícil; los niños y las niñas la utilizan como factor de distracción (videojuegos). Sobre este tema, las respuestas de una mamá se enfocaron en mencionar que desde que tiene celular ha cambiado el comportamiento de su hijo debido a que prefiere estar en cuarto que ir a jugar con sus amistades como antes lo hacía.

Sobre este tema, Vangelisti (2004) menciona que existen diferentes efectos de "la tecnología en el actuar de la familia, en un primer momento se resalta la brecha digital entre los integrantes para el uso de los aparatos, otro factor determinante es lo referente a la disponibilidad de Internet” (p. 26).

En ese sentido, Vangelisti (2004) enfatiza que:

No hay todavía investigaciones sobre los efectos que la saturación de medios tecnológicos en los hogares puede causar sobre la comunicación en la familia. Consideran que hay mucha especulación sobre el impacto de los últimos medios tecnológicos sobre la vida familiar y poca investigación. Ya que muchos de los nuevos medios tecnológicos, como sucedió con la televisión o la radio, están siendo utilizados en familias con hijos, sería necesario conocer las funciones que esos medios tienen en el mantenimiento de las relaciones familiares, en la socialización de los niños, y en la participación de las familias en la vida social fuera del hogar (p. 27).

\section{JUEGOS TRADICIONALES: PROPUESTA PARA LA MEJORA DE LA CONVIVENCIA FAMILIAR}

Durante la intervención docente desde la Educación Física, se utilizó una estrategia denominada juegos tradicionales, la cual se sustenta en los postulados teóricos de Spencer sobre el excedente de energía y de Buytendijik acerca de la teoría de la dinámica infantil (como se cita en Gallardo-López y Gallardo-Vázquez, 2018). Se destaca que los juegos tradicionales son entendidos como actividades lúdicas que se realizan por tradición y que son transmitidos de generación en generación. Por tanto, al reconocer el valor del juego como estrategia pedagógica, su uso fue de utilidad para promover la interacción entre madres, padres, hijas e hijos en ambos contextos: escolar y familiar.

La Secretaría de Educación Pública (SEP) de México afirma que los juegos tradicionales “son caracterizados por ser anónimos, y universales, pues es posible encontrarlos en todas partes del mundo" (SEP, 2006, p. 343) y en el programa de Educación Física para Primaria en México, se asegura que uno de los propósitos del juego tradicional es "reconocer la diversidad y valorar la identidad nacional, de tal forma que los juegos tradicionales y autóctonos constituyan una parte para la comprensión de la interculturalidad”, (SEP, 2011, p. 199). Además, para fortalecer un estilo de vida saludable en el alumnado, se sugiere realizar actividades pedagógicas con madres y padres de familia para promover la convivencia familiar, con el único propósito de propiciar un ambiente de sano esparcimiento entre todas las personas que conforman la comunidad (SEP, 2011).

Sobre este tema, Morena (2008) afirma que "los juegos tradicionales dan la posibilidad de que cada persona construya su juguete poniendo en práctica su ingenio y creatividad para utilizar el material disponible a su alcance, convirtiendo al niño en artesano” (p. 4). En México existen diversos juegos y juguetes tradicionales producto de la mezcla de influencias que actuaron sobre la formación de la cultura criolla de este país, algunos de ellos requieren algún tipo de objeto o material para su realización y otros no. La Revista Don Quijote (2020) detalla cada uno de estos juegos que han divertido a la población mexicana a través de los años: a) juegos tradicionales con objetos: pirinola, yoyo, trompo, papalote, carrera con sacos, saltar la cuerda, la papa 
se quema, el palo encebado, las canicas y los hoyitos; b) juegos tradicionales sin objetos: el avión, las estatuas, las rondas y el stop. En la Figura 2, se puede observar a un grupo jugando canicas.

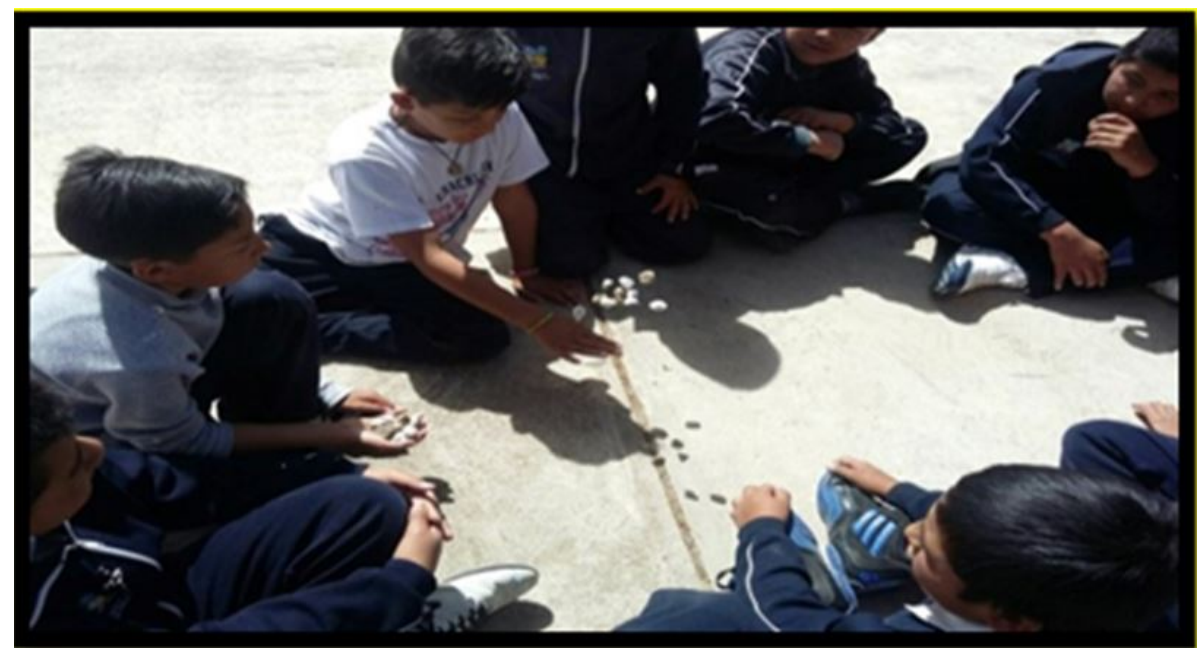

FIGURA 2.

Juego tradicional las canicas

Fuente: colección de la autoría.

No obstante, los juegos tradicionales han perdido relevancia en la familia debido a la falta de tiempo de los padres y madres para jugar con sus hijos e hijas por los cinco factores descritos. Un aspecto importante es la falta de tiempo de dedicación a los hijos e hijas, la comunicación y la escasa práctica hacen que estos juegos no sean divertidos para la población infantil.

Los juegos tradicionales se llevaron a cabo en diez sesiones de educación física, en la que se aplicaron 9 juegos (cuerda, matatena, stop, circuito tradicional, hoyitos, yoyo, canicas, balero y trompo); en la última sesión se realizó una feria de juegos tradicionales denominada Los juegos de mi abuelo. La feria de juegos tradicionales fue una estrategia para observar el comportamiento de las familias en la escuela y verificar si los juegos realizados en la sesión de educación física fueron jugados en casa con el objetivo de convivir en familia. En la Figura 3 se puede apreciar la ficha utilizada para dar seguimiento a los juegos realizados.

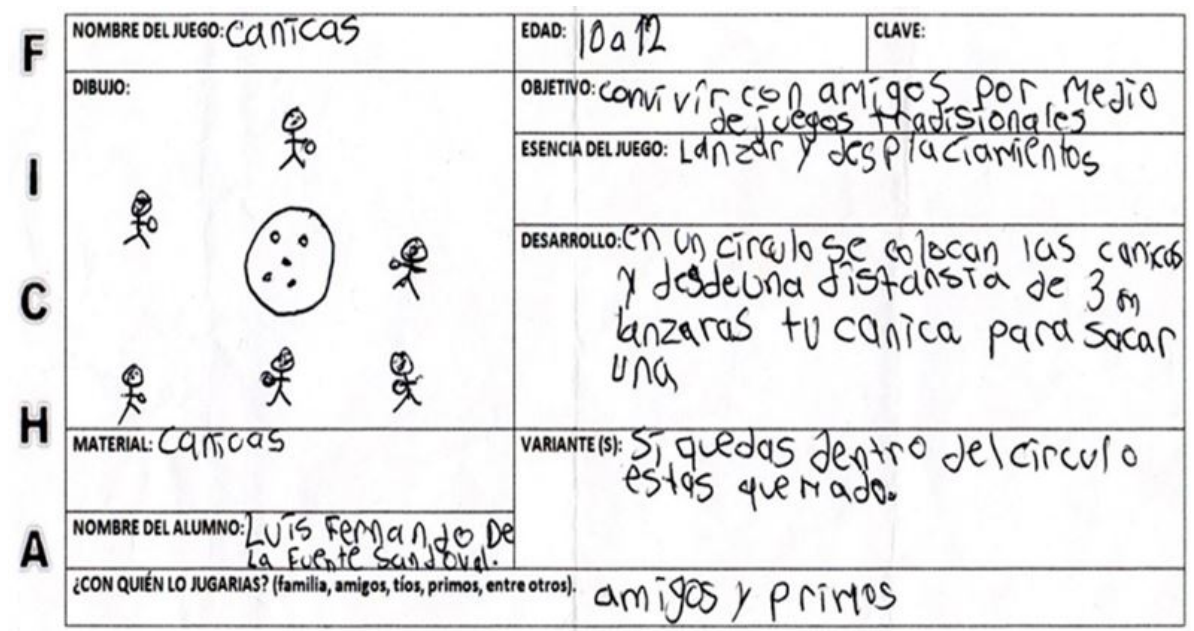

FIGURA 3.

Ficha del juego tradicional "las canicas"

Fuente: colección de la autoría.

Se contó con la colaboración del alumnado de otros salones para que monitorearan y enseñaran el juego al equipo que llegara. Aún con los constantes recordatorios sobre la actividad, algunos padres y madres de 
familia no llegaron por diferentes motivos, pues solo llegaron 20 personas a acompañar a al estudiantado y no solo era la mamá, también la tía, la vecina o la madrina. Solo un alumno trajo a su familia completa (mamá, papá y hermano), quienes se divirtieron mucho. Se organizó un circuito de acción motriz, pues tenían que pasar a cada estación, toda dentro de un resorte (abrazando a la familia), en cada juego hubo expresiones diferentes.

En la actividad de la cuerda, los niños y las niñas saltaban, los papás y mamás solo tomaban la cuerda, pues algunos tenían problemas físicos, y las abuelitas, por obvias razones, no podían, o las mamás que llevaban falda; en la estación de serpientes y escaleras, con la actividad de mesa tradicional, pudieron enfrentarse y disfrutar del juego.

En el avión, además de no tener equilibrio, los papás y mamás no sabían cómo se jugaba, pues se les hizo poco agradable; en el stop, juego de mucha alegría y entusiasmo, se observó la actitud positiva de los padres y madres, como fueran los niños o las niñas; el toro congelado, juego que ya conocían, pero aun así se realizó de manera divertida e implementó la velocidad de reacción para evitar ser tocadas o tocados.

En la estación de carrera de costales, al igual que la cuerda, los padres y madres de familia no querían saltar con el costal hasta el otro extremo por diferentes motivos: me duele las rodillas, están lastimadas, algunos no traían la ropa adecuada, también influía la edad de la madre o padre, y algunos abuelos; pero no fue motivo para no apoyar a que las niñas y niños a participar y saltar en lugar de sus madres y padres; la estación del trompo y yoyo resultó difícil para las niñas y niños y fácil para las personas adultas, pues esos fueron sus primeros juguetes cuando eran niños o niñas, con los que pasaron horas jugando, pero los niños y niñas se desesperaron porque no podían realizarlo; la estación de lotería fue un juego de mucha alegría y entusiasmo por parte de los padres y madres, pues tenían que poner mucha atención para escuchar y observar las figuras que tenían para poder ganar. Ver Figura 4.

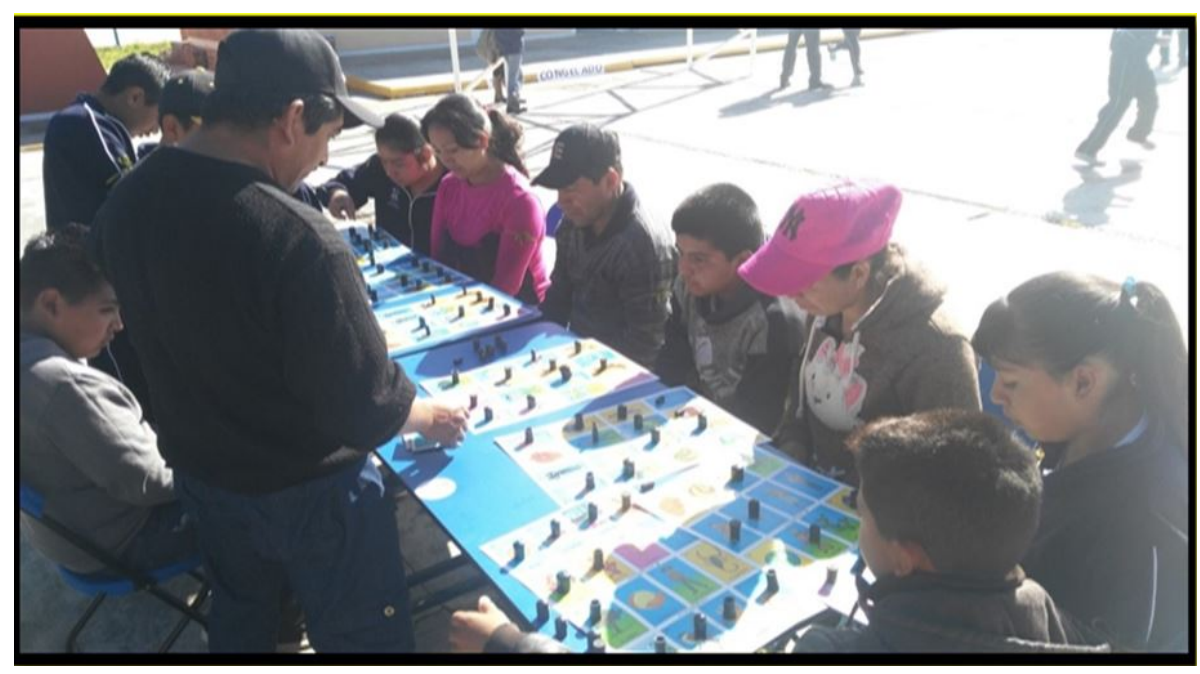

FIGURA 4.

Feria de juegos tradicionales: estación la lotería

Fuente: colección de la autoría.

Definitivamente es importante rescatar este tipo juegos, porque actualmente la población infantil está muy allegada a la televisión, al uso de equipos electrónicos y se pierde la experiencia formativa de la interacción social con la familia.

Por tanto, las sesiones de educación física con el grupo de estudio fueron de utilidad para verificar la realización de los juegos tradicionales con la familia. A partir de las fichas de juegos entregadas por el alumnado, se diseñó una gráfica (Figura 5), la cual se puede apreciar abajo. 


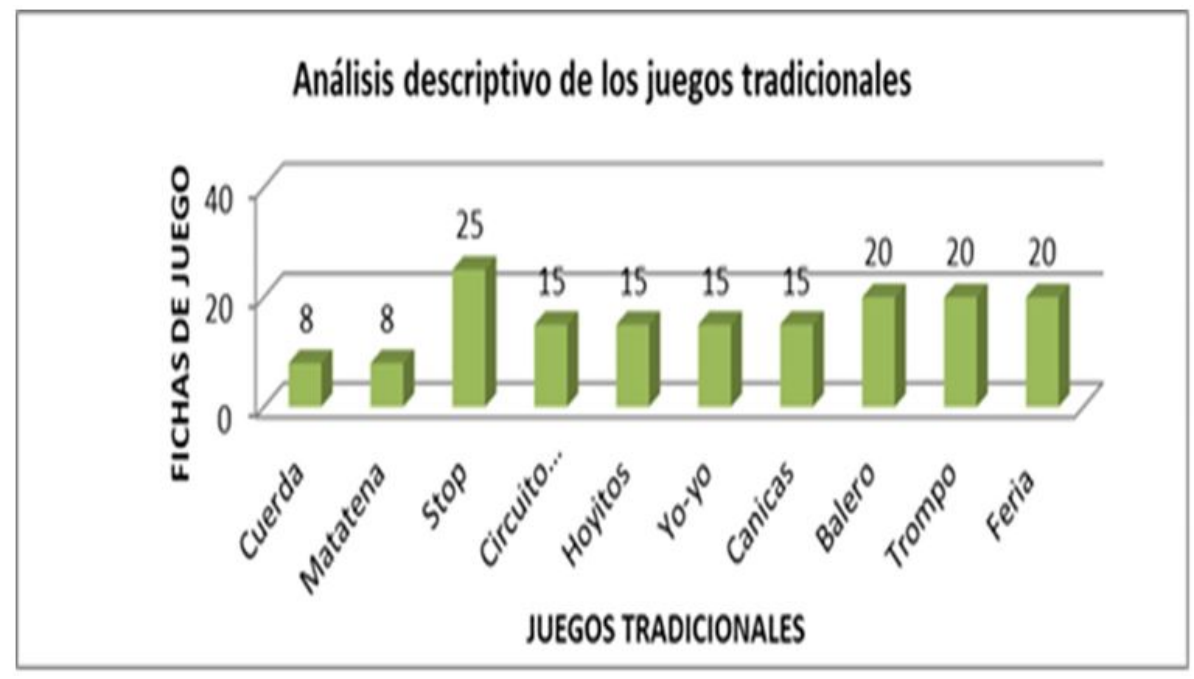

FIGURA 5.

Análisis descriptivo de los juegos tradicionales realizados en casa

Fuente: elaboración propia.

\section{Conclusiones}

Los resultados de la investigación determinan que los factores que afectan la convivencia entre padres, madres, hijos, hijas de la comunidad en estudio son: 1) escasa comunicación, 2) limitado tiempo libre compartido, 3) escaso interés de los padres y madres con el rendimiento escolar de sus hijos e hijas, 4) responsabilidad laboral de los padres y madres de familia, y 5) uso excesivo de aparatos electrónicos, principalmente por parte del alumnado (TV, Smartphone e Xbox).

La convivencia entre madres, padres, hijas e hijos no es la adecuada. Se pudo observar que a los padres y madres de familia no les interesaba ir a la escuela, ni a reuniones convocadas por la institución o por la maestra de grupo, por motivos de tiempo (según los padres o madres), de trabajo laboral o simplemente por descuido. Se constató que existían problemas en la comunicación y relaciones de familia, específicamente entre papá y mamá, esto repercutía en la conducta y actitud del niño o de la niña, tanto dentro como fuera de clases.

El uso de los juegos tradicionales en las sesiones de educación física, las tareas realizadas mediante las fichas y, principalmente, la feria de juegos tradicionales, fueron actividades que propiciaron la interacción y mejoraron el vínculo afectivo de padres y madres de familia con sus hijos e hijas.

El uso de juegos tradicionales en la clase de educación física provocó entrar en contacto con las familias: se establecieron lazos afectivos y se compartieron inquietudes, éxitos, amor y metas.

De forma gradual, la mayoría del alumnado y sus familias realizaron tareas encomendadas: no faltar a clases, tener una actitud positiva en la realización los juegos tradicionales, participar dentro y fuera de la escuela, mantenerse en contacto con el personal docente del centro educativo en donde estudia su hijo o hija y estar cada vez más al pendiente de su desempeño.

Utilizar los juegos tradicionales como estrategia pedagógica ayudó a entrar en contacto con la familia. Mediante la invitación a participar en la feria de juegos tradicionales, la familia del estudiantado logró integrarse y comprometerse a trabajar en sintonía con la escuela, al reconocer lo que sus hijos e hijas realizan en las sesiones de Educación Física. Por tanto, el uso de los juegos tradicionales contribuyó en la mejora del ambiente social.

Los juegos tradicionales contribuyeron a mejorar la convivencia familiar del alumnado de quinto grado, el cual se sentía motivado por aprender estos juegos y repetirlos en casa, además de convivir con su familia. Un aspecto importante fue el interés y motivación de las madres y padres, quienes se involucraron poco a poco 
al preguntar por el avance y desempeño que tiene sus hijas e hijos en la escuela; así como atender a llamados, participar en actividades de la institución y en las rutinas de activación masivas.

La influencia de los juegos tradicionales en la sesión de Educación Física y tareas extraescolares fue de gran utilidad para fomentar la convivencia familiar. Puesto que hechos tan simples como que las madres y padres dijeran a sus hijos e hijas buenos dias o te amo y estuvieran al pendiente, favorecen la convivencia.

Se pudo destacar aspectos que no favorecen la convivencia familiar; tales hallazgos pueden utilizarse para contribuir a la mejora. Las buenas relaciones, así como la comunicación que existe en las familias, produjo sentimientos, sensaciones y percepciones de culpa, resentimiento, felicidad, compañerismo, inquietud y una meditación de lo que está pasando en cada familia.

Con los resultados obtenidos en la investigación se considera que debe de estudiarse más a fondo lo siguiente:

a) Hasta qué grado la responsabilidad laboral es importante en la familia. Puesto que en la investigación algunos padres y madres de familia le daban demasiada importancia al sustento familiar. Evidentemente, este aspecto es básico, pero se considera que por ese motivo se descuida el lado humano al no estar con el niño o la niña de manera física y emocional. Por ello, se recomiendan talleres de parentalidad positiva, en donde los padres y madres de familia reconozcan la gran labor que tienen para la formación de personas felices.

b) ¿Qué influencia tiene la tecnología en los preadolescentes? La tecnología, como bien se ha descrito, no es una mala influencia en los niños o niñas, al contrario, les ayuda en su aprendizaje, siempre y cuando esta sea utilizada de manera correcta.

c) ¿Cómo involucrar a cada integrante de la familia en el desarrollo académico de la población infantil? Lo anterior se enfocaría a identificar las causas que provocan ese desinterés de los padres y madres por el aprendizaje de sus hijos o hijas. Sobre este tema, una madre de familia comentó que no tiene tiempo de ir a la escuela: otro día voy a ver de qué se trató la reunión, esos maestros siempre nos citan, pero que no saben que tenemos cosas que hacer; estas frases que utilizan para no ir a la escuela, en especial para preguntar cómo va su hijo o hija para darle apoyo en el proceso de aprendizaje. Por ello, se percibe la necesidad de implementar más proyectos que favorezcan el involucramiento de la familia en la formación integral de los niños o niñas mediante juegos, para aprovechar la dinámica infantil de interés por el juego y el excedente de energía en actividades lúdicas.

Por tanto, se sugiere la implementación de los juegos tradicionales en el entorno escolar y familiar, debido a los beneficios que estos aportan, desde el aspecto cultural, la interacción social, el gasto de excedente de energía y, primordialmente, el aprovechamiento de la dinámica infantil y el gusto por el juego. Además, el juego tradicional es valioso como instrumento para favorecer el vínculo afectivo entre las personas que integran la familia; además de que es el principal recurso pedagógico en la escuela, que bien puede ser llevado a casa para que el estudiantado ejerza su derecho al juego y los padres y madres puedan manifestar esa actitud o dinámica infantil para encauzar el excedente de energía de sus hijos o hijas mediante actividades lúdicas.

\section{REFERENCIAS}

Álvarez-Gayou, J.L. (2003). Cómo hacer investigación cualitativa: fundamentos y metodología. Paidós.

Bowlby, J. (1986). Vinculos afectivos. Formación, desarrollo y pérdida. Morata.

Creswell, J. (2005). Educational Research: Planning, Conducting, and Evaluating Quantitative and Qualitative Research. Internacional edition.

De León, B. (2011). La relación familia y su repercusión en la autonomia y responsabilidad de los niños. INDE.

Desarrollo Integral de la Familia [DIF]. (2020). Sistema Estatal DIF. Gobierno de Puebla. http://difestatal.puebla.g ob.mx/ 
Gallardo-López, J. A. y Gallardo-Vázquez, P. (2018). Teorias del juego como recurso educativo [Conferencia]. IV Congreso Virtual Internacional sobre Innovación Pedagógica y Praxis Educativa. https://rio.upo.es/xmlui/han dle/10433/6824

Hernández, M. A. y Carrión, E. (2019). Formación inicial de los maestros de educación infantily las conductas disruptivas de la convivencia escolar [Conferencia]. IV Congreso internacional sobre la educación en el Siglo XXI. https:// www.eumed.net/actas/19/educacion/1-formacion-inicial-de-los-maestros.pdf

Hernández, R., Fernández, C. y Baptista, P. (2014). Metodología de la investigación. (6ta edición). McGraw Hill.

Lillo, J. y Leiva, V. (2012). Importancia de la familia para la convivencia escolar. Universidad del Bio-Bio. https://lice ntiare.wordpress.com/2012/01/18/importancia-de-la-familia-para-la-convivencia-escolar/

Loizaga, F. (2011). Parentalidad positiva. Las bases de la construcción de la persona. Revista Educación Social, (49), 70-88. https://www.raco.cat/index.php/EducacioSocial/article/view/250179

Megías, E. y Elzo, J. (2006). Jóvenes, valores y drogas. EDIS. https://www.fad.es/wp-content/uploads/2019/05/valo res06.pdf

Massoi, I., Dorio, I. y Sabariego, M. (2009). Estrategias de recogida y análisis de la información. En R., Bisquerra. Metodología de la investigación educativa (pp. 229-365). Editorial La Muralla.

McMillan, J. y Schumacher, S. (2011). Investigación educativa. Edición Pearson Educación.

Meece, J. (2001). Desarrollo del niño y del adolescente. SEP/ULTRA.

Morena, M. (2008). Generación tras generación, recobran los juegos tradicionales. Revista en Ciencias del Movimiento Humano y Salud, 5(1) 1-8. https://www.redalyc.org/pdf/2370/237017527002.pdf

Murillo, E. M. (2009). Estrategias para la integración de las familias en el mejoramiento de los aprendizajes escolares [Tesis doctoral]. Universidad Nacional de Educación a Distancia. http://repositorio.minedu.gob.pe/handle/2 $0.500 .12799 / 1830$

Ramírez-García, A., González-Fernández, N. y Salcines-Talledo, I. (2018). Competencias parentales positivas y Smartphones. Diagnóstico en el contexto familiar. Revista Mediterránea de Comunicación/Mediterranean Journal of Communication, 9(2), 137-157. https://rua.ua.es/dspace/bitstream/10045/76872/1/ReMedCom_ 09_02_28.pdf

Revista Don Quijote. (2020).Juegos tradicionales de México. https://www.donquijote.org/es/cultura-mexicana/trad iciones/juegos/

Rodrigo, M. J. (2015). Manual práctico de parentalidad positiva. Editores Madrid Síntesis. https://dialnet.unirioja.es /servlet/libro?codigo $=570396$

Rodríguez, G., Gil, J. y García, E. (1999). Metodología de la investigación cualitativa. (2da edición). Ediciones Aljibe. Seligman, M. (2017). La auténtica felicidad. Ediciones B.

Secretaría de Educación Pública [SEP]. (2006). Programas de Educación Física. SEP.

Secretaría de Educación Pública [SEP]. (2011). Programas de Educación Física. SEP.

Vangelisti, A. L. (2004). Handbook offamily communication. Lawrence Erlbaum. https://pdfs.semanticscholar.org/4 688/10659fbb4a29552a97e051f581739f190b34.pdf

\section{INFORMACIÓN ADICIONAL}

Cómo citar: Coyotecatl-Xochimitl, L. M. y Murrieta-Ortega, R. (2022). Factores que afectan la convivencia familiar: análisis micro etnográfico a través de juegos tradicionales en un grupo social en México. Revista Educación, 46(1). http://doi.org/10.15517/revedu.v46i1.43829 